\title{
Publikationen von Prof. Dr. med. Erwin H. Ackerknecht
}

Fortsetzung der Liste in Gesnerus 1/2 (1966), die bis 1965 reicht

\section{Bücher}

Medicine at the Paris Hospital 1794-1848, Johns Hopkins Press, Baltimore 1967.

Therapie von den Primitiven bis zum 20. Jahrhundert, Enke, Stuttgart 1970.

Medicine and Ethnology, Huber, Bern 1971.

Kurze Geschichte der großen Schweizer Arzte (mit Heinrich Buess), Huber, Bern 1975.

\section{Übersetzung}

Ambroise Paré, Rechtfertigung und Bericht über meine Reisen in verschiedene Orte. Hubers Klassiker der Medizin und der Naturwissenschaften, Band 2, Huber, Bern 1963.

\section{Artikel}

1965 Diseases in the Middle West. D.J.Davis Mem., Chicago 1965, S. 168-181

1966 Medizinische Dokumente aus Großmutters Mappe. Image, Nr.17, 1966, S. 12-17

La médecine à Genève surtout dans la première moitié du XIX ${ }^{\mathrm{e}}$ siècle. C.R. XIX Congr.int. Histoire de la Médecine, Basel 1964, Karger, Basel 1966, S.420-425

Zur Geschichte der Tollwut. Schweiz.Med.Wschr.96 (1966) $746-748$

Vorwort zu: Wilhelm Griesinger, Mental Pathology. Reprint, New York 1966

Vorwort zu: Theodor Puschmann, History of Medical Education. Reprint, New York 1966

Charles Nicolle. Die Welt, 21.Sept. 1966

A hundred years of American Medicine 1866-1966. Intern. Forum 14. (1966) 127-130 
Rudolf Virchow. In: René Dumesnil und Hans Schadewaldt (Hrsg.), Die berühmten Ärzte, Köln 1966, S.225-226

Grands médecins suisses 1500-1900. Confér. Palais de la Découverte D 109, Paris 1966, 24 S.

Introduction to a bibliography of the writings of Henry E. Sigerist, Montreal 1966, S.1-7

1967 Einleitung zu: Huldrych M. Koelbing, Renaissance der Augenheilkunde 1540-1630, Huber, Bern 1967, S.9-10

Zur Geschichte des Ikterus. Praxis 56 (1967) 282-283

Der Tod in der Geschichte der Medizin. VESKA 31 (1967) 230-231

A Plea for a "Behaviorist" Approach in writing the History of Medicine. J.Hist. Med.22 (1967) 211-214

1968 Zellulartheorie und Therapie. Praxis 57 (1968) 126-127

Death in the History of Medicine. Bull. Hist. Med.42 (1968) 19-23

Introduction to: H. R.Isler, Thomas Willis, New York 1968, S. VII-VIII

Der letzte Geheimrat. Tages-Anzeiger Zürich, 29. Juni 1968, S. 18

The Vagaries of the Notion of Epidemic Hepatitis. Historical Essays in Honor of Owsei Temkin, Baltimore 1968, S.3-16

Einleitung zu: Jean Etienne Dominique Esquirol, Von den Geisteskrankheiten, Huber, Bern 1968, S. 7-10

Das Märchen vom verlorenen Psychosomatismus. Gesnerus 25 (1968) 113-115

Mediziner und Zellenlehre. Gesnerus 25 (1968) 188-194

Zum 100.Geburtstag von Max Neuburger. Gesnerus 25 (1968) 221-222

Vorwort zu: J.H. Donhoff, Der Arzt und sein Honorar im Wandel der Zeit, Zürich 1968, S.7

1969 Therapeutische Selbstversuche. Documenta Geigy 1969: Ärzte als Patienten und Versuchspersonen, S.5-6

Die Therapie im Fegefeuer während des 19. Jahrhunderts. Österreichische Arztezeitung 24 (1969) 521-526

Die therapeutische Erfahrung und ihre allmähliche Objektivierung. Gesnerus 26 (1969) 26-35

Ambroise Paré. Image, Nr.32, 1969, S.26-32 
1970 A short survey of Drug Therapy prior to 1900. In: Blake ed., Safeguarding the public, Baltimore 1970, S.51-58

Therapie und therapeutische Moden vor fünfzig Jahren. Praxis 59 (1970) 306-308

Cellular Theory and Therapeutics. Clio Medica 5 (1970) 1-5

Nonideological elements in the History of Psychiatry. In: Mora ed., Psychiatry and its History, Springfield 1970, S.223-228

Zur Geschichte der iatrogenen Erkrankungen des Nervensystems. Therapeutische Umschau 27 (1970) 345-346

David d'Angers und seine Medaillons zeitgenössischer Mediziner. Image, Nr. 37, 1970, S.28-32

Zur Geschichte der medizinischen Sekten. Documenta Geigy 1970, S. 5-6

Zur Geschichte der iatrogenen Krankheiten. Gesnerus 27 (1970) $57-63$

Zurich theses. Journ. Hist. Behav.Sc.6 (1970) 269-270

"The Discovery of the Unconscious". Neue Zürcher Zeitung, 25. Okt. 1970, S.53

«Das Hippokratische Ethos». Schweizerische Arztezeitung 51 (1970) 834-835

Die Zürcher Schwangerschaftsuhr. Zürcher Album, hrsg. von U. Isler, Zürich 1970, S.76-77

Zum hundertsten Geburtstag von Fielding H. Garrison. Gesnerus 27 (1970) 229-230

Vorwort zu: Esther Fischer-Homberger, Hypochondrie, Huber, Bern 1970, S.9-10

1971 Holland im 17. Jahrhundert: medizinisch und kulturell. Gesnerus 28 (1971) 1-6

Zum 60. Geburtstag von Heinrich Buess. Gesnerus 28 (1971) 79-80

Erna Lesky. Österreichische Ärztezeitung 26 (1971) 1076

Manieristische Anatomie: Frederik Ruysch und seine Sammlung. Image 1971, S.27-32

Suchtprobleme im Lauf der Jahrhunderte. Praxis 60 (1971) 10061009

Jüdische Ärzte als Gestalter der Weltmedizin. Vortrag gehalten am 25. November 1970 in der Jüdischen Vereinigung, Zürich. Privatdruck 1971, $9 \mathrm{~S}$. 
The end of Greek diet. Bull. Hist. Med.45 (1971) 242-249

Die Geschichte der medizinischen Beziehungen zwischen der Schweiz und den USA. Verhandlungen der Schweizerischen Naturforschenden Gesellschaft, Wissenschaftlicher Teil, 151.Jahresversammlung vom 8.-10.0ktober 1971 in Freiburg, Birkhäuser, Basel, S. 26-32

1972 Medizinische Praxis im alten Zürich. Neue Zürcher Zeitung, 30. Jan. 1972

Vorwort zu: J.H. Donhoff, Ärzte aus Forschung und Praxis in der deutschsprachigen Schweiz, St. Gallen 1972, S.5-7

Vorwort zu : Cécile Ernst, Teufelsaustreibungen, Huber, Bern 1972, S. 7

The History of medical relations between Switzerland and the USA. Gesnerus 29 (1972) 69-77

In Memoriam Richard H.Shryock. Clio Medica 7 (1972) 129-130

Der Deutsch-Französische Krieg 1870/71 in der Graphik. Image, Nr. 50, 1972, S. 34-40

Akupunktur - gestern, heute, morgen. Schweizerische Ärztezeitung 53 (1972) 1067-1068

Akupunktur - eine Antwort an ihre Verteidiger. Schweizerische Arztezeitung 53 (1972) 1543-1544

1973 Der Erste Weltkrieg in der französischen Graphik. Image Roche, Nr. 52, 1973, S. 33-40

Der Erste Weltkrieg in der deutschen Graphik. Image Roche, Nr. 53, 1973, S. 33-40

Medizin und öffentliche Gesundheitspflege in der Chinesischen Volksrepublik. Neue Zürcher Zeitung, 28. Mai 1973, S. 33

Boerhaave-Schüler als Medizinalpolitiker. In: Gerard van Swieten und seine Zeit (hrsg. von Erna Lesky), Wien 1973, S.121-127

Benedict Stilling (1810-1879). Gesnerus 30 (1973) 143-149

Medicina y sociedad en la ilustración. In: Pedro Lain Entralgo, Historia universal de la medicina, Barcelona 1973, vol. 5, S. 143-151

In memory of Suessman Munter. Clio Medica 8 (1973) 241

1974 Zur Geschichte der Akupunktur. Anaesthesist 23 (1974) 37-38

The History of the Discovery of the Vegetative (Autonomic) Nervous System. Med.Hist. 18 (1974) 1-8 
Die Holzschnitt-Porträts von Felix Vallotton. Image Roche, Nr.60, 1974, S. 29-33

Die ersten 100 Jahre des Kinderspitals Zürich. In: 100 Jahre

Kinderspital Zürich 1874-1974, Zürich 1974, S.37-48

Zur Geschichte der Hebammen. Gesnerus 31 (1974) 181-192

Une vieille histoire. Médecine et Hygiène 32, No 1118 (Histoire de la médecine), 9 octobre 1974, S. 1571

1975 Von den Ärzten und der «Kunst» der Euthanasie. Medical Tribune 8 (1975) 9

Klassenkampf am Krankenbett. Schweizerische Ärztezeitung 56 (1975) 483-486; Nieders. Ärzteblatt 1975, No. 8, S.241-243; Brem. Arzteblatt 28, 10 (1975) 9-14

Aus den Anfängen der Neurochirurgie. Schweizer Archiv für

Neurologie, Neurochirurgie und Psychiatrie 116 (1975) 233-239

Political prisoners in French mental institutions before 1789, during the Revolution, and under Napoleon I. Med. Hist. 19 (1975) $250-255$

Medizin und Karikatur. Du, Nr.10, Okt.1975, S.66-73

Rudolf Virchow und die Sozialmedizin. Sudhoffs Archiv 59 (1975) 247-253

Brief (concerning C. G. Jung). J. Hist.Behav.Sc.11 (1975) 409

Erich Hintzsche gestorben. Gesnerus 32 (1975) 227

Zur Geschichte der Arteriosklerose. Gesnerus 32 (1975) 229-234

1976 Geriatriegeschichtliches. Praxis 65 (1976) 320-323

\section{Buchbesprechungen im «Gesnerus»}

Die 41 Buchbesprechungen, die Ackerknecht 1958-1968 im «Gesnerus» veröffentlicht hat, sind im «Register der Jahrgänge 1 bis 25 », S.5-7 zusammengestellt. 1969-1975 sind 57 weitere erschienen. 